\title{
Excision biopsy of malignant melanoma by general practitioners in south east Scotland 1982-91
}

\author{
R M Herd, J A A Hunter, K M McLaren, U Chetty, A C H Watson, J M Gollock
}

Variables analysed

Sex

Age

Longest diameter

Histogenic type

Breslow thickness

Clearance

Time to wider re-excision
University Departments of

Dermatology and

Pathology, Royal

Infirmary, Edinburgh

EH3 9YW

$\mathrm{R} M$ Herd, registrar in

dermatology

J A A Hunter, professor of

dermatology

K M McLaren, senior lecturer

in pathology

Department of Surgery, Western General Hospital, Edinburgh

U Chetty, consultant surgeon

Plastic Surgery Unit, St John's Hospital, Livingston A C H Watson, consultant plastic surgeon

Department of Surgery, Borders General Hospital J M Gollock, consultant surgeon

Correspondence to: Dr Herd.

\section{Abstract}

Objective-To examine the management of patients who had a malignant melanoma excised initially by general practitioners in south east Scotland over the past 10 years and to assess the impact of the April 1990 contract on this.

Design-A retrospective case-control study.

Setting-South east Scotland.

Subjects-All patients in south east Scotland who had malignant melanomas excised by general practitioners in 1982-91.

Outcome measures-Demographic details of patients; Breslow thickness, clearance of excision.

Results-42 patients had malignant melanomas excised by general practitioners in 1982-91: 15 in $1982-9$ and 27 in 1990-1. These patients were significantly younger than those who had their tumours excised initially in hospital. Although the longest diameter of melanomas excised by general practitioners was significantly less than of those excised in hospital, the Breslow thicknesses were similar. Completeness of initial excision was doubtful or incomplete in nine $(23 \%)$ general practitioner excisions compared with $4 \%$ of hospital excisions, but the time interval between excision biopsy and wide excision was similar. Pathology requests accompanying excision biopsies mentioned melanoma as a possible diagnosis in $15 \%(6 / 40)$ of general practitioner cases compared with $79 \%$ of hospital cases. Thirty nine general practitioners responded to a questionnaire and only 12 had considered melanoma in the differential diagnosis.

Conclusions-General practitioners need to think more often of malignant melanoma when they excise pigmented lesions and when they consider this tumour a possibility should perform an excision biopsy with a lateral clearance of at least $2 \mathbf{~ m m}$.

\section{Introduction}

When the new general practitioner contract was introduced in the United Kingdom on 1 April 1990 general practitioners on the minor surgery list became eligible for a fee for carrying out certain minor surgical procedures, including skin biopsies.

Skin biopsy specimens submitted by general practitioners to pathology departments have increased at least fourfold since the contract, without an accompanying reduction in those submitted from hospital practitioners. $^{2-5}$ Worries have also been expressed about diagnostic accuracy and the completeness of excision of malignant lesions. ${ }^{236}$ These earlier studies included a small number of malignant melanomas. As the incorrect diagnosis of malignant melanomas can have serious consequences we looked separately at excisions of malignant melanomas by general practitioners to identify areas where management could be improved.

\section{Methods}

The data used for this study came from the malignant melanoma registrations in south east Scotland held on the computerised records of the Scottish Melanoma Group. All patients registered who had had malignant melanomas excised initially by general practitioners over the past 10 years were included in the study.

For each patient two controls were randomly selected from among patients who had had excisions carried out in hospital over the same period, the number of controls in each year being weighted according to the numbers of general practitioner excision biopsies in that year.

All general practitioner excisions were primary narrow excisions and were compared only with similar excisions carried out in hospital. Those who had primary wide excisions in hospital and those who were judged not to require a subsequent wider re-excision were excluded from the study. The variables recorded are shown in the box and, using these, we compared primary excisions carried out in hospital with those carried out by general practitioners; these were also compared before and after the April 1990 contract. The original pathology requests, completed when the primary excision was carried out, were also examined for the mention of "mulanoma" in the differential diagnosis.

To gather further information about general practitioners' attitudes to excision and management of malignant melanoma we sent a questionnaire to all general practitioners who had excised a malignant melanoma during the study period. This asked why the patient attended and why the lesion was excised; the provisional diagnosis; the interval between the patient first consulting and the excision; and whether the general practitioner's attitude towards excising pigmented lesions had changed. Two general practitioners whose patients had subsequently developed metastases were excluded.

The statistical tests used were the $\chi^{2}$ test with Yates's correction and the $t$ test, which was carried out following logarithmic transformation when indicated.

\section{Results}

In 1982-91, 42 malignant melanomas were excised by general practitioners in south east Scotland (table). Forty eight per cent (20) were excised from men, a proportion not significantly different from the ratio found among hospital excisions $(36 \% ; 30 / 84)$. The mean age of those with malignant melanoma excised initially by general practitioners was 41 years, significantly less than the mean age among patients with hospital excisions, 51 years $(p<0.01)$.

No firm conclusions could be drawn from the distribution of the anatomical site of the malignant melanomas excised by general practitioners; because 


\begin{tabular}{lr}
\hline Year & No \\
\hline 1982 & 0 \\
1983 & 0 \\
1984 & 2 \\
1985 & 4 \\
1986 & 0 \\
1987 & 0 \\
1988 & 4 \\
1989 & 5 \\
1990 & 16 \\
1991 & 11 \\
\hline
\end{tabular}

the numbers were small the distribution did not differ significantly from that found among hospital excisions.

Among the 42 malignant melanomas excised by general practitioners were 33 superficial spreading melanomas, eight nodular melanomas, and one acral lentiginous melanoma. This ratio did not differ significantly from that of those excised initially in hospital

The distribution of the longest diameters of the malignant melanomas was significantly skewed to the right. The data were therefore logarithmically transformed before being analysed. The mean diameter of malignant melanomas excised by general practitioners was $6.1 \mathrm{~mm}$, which was significantly less than that among hospital excisions, $9.7 \mathrm{~mm}(\mathrm{p}<0.001)$. The Breslow thickness of general practitioner and hospital excisions was not significantly different, with about $18 \%$ of both groups having a Breslow thickness $>3.0 \mathrm{~mm}$.

When the excision biopsy is examined by the pathologist the completeness of excision is assessed as complete, incomplete, or doubtful. This information is contained in the database of the Scottish Melanoma Group. Ten general practitioner excisions were classified as incomplete or doubtful compared with only three hospital excisions $(p<0.001)$.

The time interval between the primary narrow excision and the wider re-excision was skewed to the right so this variable was analysed in a similar way to the longest diameter. The mean time to re-excision for general practitioner biopsies (35 days) was not significantly different from that for hospital excision biopsies (31 days).

When the original pathology requests were examined only six out of $40(15 \%)$ general practitioner request forms mentioned melanoma as a possible diagnosis compared with 66 out of $84(79 \%)$ hospital excisions.

There was no difference in any of the above variables between the period before and after the April 1990 contract.

Thirty nine general practitioners were sent questionnaires, and all replied. Of their 39 patients 25 attended because of a changing lesion, most often a change in size. Sixteen patients were worried about malignancy, while nine were concerned about cosmetic appearance. Only six were excised for cosmetic reasons alone, the remainder being excised either for suspected malignancy or to exclude malignancy. The differential diagnosis included malignant melanoma in 12 cases $(31 \%)$. The other provisional diagnoses included benign naevus ( 17 cases), squamous cell carcinoma (2), seborrhoeic keratosis (1), dysplastic naevus (1), dermatofibroma (1), haemangioma (1), and "don't know" (3).

The delay in excision biopsy from the time the patient first attended with the lesion was longer than a month only once. The median delay was seven days.

There were 37 replies to the question on attitude to future management of pigmented lesions. Twenty four general practitioners said no change, 11 had been encouraged to excise more, while only two had been discouraged by their experience.

\section{Discussion}

Although there have been studies of excisions of malignant skin lesions by general practitioners, this is by far the largest series reporting malignant melanomas excised by general practitioners in Britain, previous series having included at most four melanomas. ${ }^{26}$ Malignant melanoma is important because it continues to affect a young population and cause considerable mortality.

The average number of malignant melanomas excised by general practitioners was two a year in
$1982-9(2.5 \%$ of all malignant melanomas in south east Scotland). In 1990, the year the new contract was introduced, this number rose dramatically to 16 , which represented $12 \%$ of all malignant melanomas in south east Scotland during that year. In 1991 the proportion fell back slightly to about $9 \%$. There was therefore a nearly fourfold increase in the numbers of malignant melanomas excised by general practitioners after the new contract, which reflects findings in other studies. ${ }^{2-4}$

The diameter of a melanoma is a poor predictor of its depth, ${ }^{7}$ and our findings are consistent with this observation. Despite the longest diameter being significantly less among general practitioner excision biopsies, the distribution of depths was almost identical to that found among hospital excisions. In particular, a similar percentage of malignant melanomas occurred in the poor prognostic group (Breslow $>3.0 \mathrm{~mm}$ ) among general practitioner and hospital excisions.

ENSURING ADEQUATE MARGIN OF CLEARANCE

At present there is no compelling evidence that incisional biopsy of malignant melanoma increases the likelihood of metastases. ${ }^{89}$ Incisional and punch biopsies are occasionally performed on lesions where excision would be difficult because of their size or location-for example, nose and hands-but these are best left to specialists. Primary excision biopsy should be carried out with a minimum lateral clearance of $2 \mathrm{~mm} .{ }^{10} \mathrm{~A}$ cuff of subcutaneous fat deep to the tumour should be included in the initial excision, but the biopsy specimen should not extend down to the deep fascia as this might compromise the subsequent wide excision (usually carried out by a surgeon). This allows an accurate measurement of the maximum depth of the tumour, which is impossible with an incisional biopsy; the depth (Breslow thickness) in turn provides a guide to the margin of clearance required for delayed wide re-excision. It is therefore important that all practitioners excising pigmented lesions ensure that an adequate margin of clearance is achieved at the time of initial excision biopsy.

\section{DELAY}

There is an inevitable delay between excision biopsy and delayed wide excision, attributable to the time for the pathology results to be reported, the patient informed, the further appointment made, and the procedure carried out. Reassuringly, the time from excision biopsy to wider re-excision was no longer for general practitioner excisions than for hospital excisions, with means of 35 and 31 days respectively. Landthaler et al provided evidence that patients undergoing narrow excision biopsy followed by delayed wide excision showed a five year disease free interval and five year survival similar to that of patients who had primary radical treatment." They also compared a delay before wide excision of less than 21 days with a delay of more than 21 days and showed no difference in outcome. Lederman and Sober also showed that re-excision within 30 days was a safe procedure associated with no reduction in survival. ${ }^{12}$ Despite the implication that a delay of up to 30 days does not influence survival general practitioners and hospital practitioners should be encouraged to reduce this delay to the absolute minimum by continuing to review communication between the departments concerned.

In Edinburgh the pathologists contact the general practitioner by telephone as well as issuing a report. By this means, a discussion of the particular lesion ensues and some advice is given about the subsequent referral for wider excision. Such referral would depend on Breslow depth, anatomical site, and particular factors relating to the individual patient, but this close 
cooperation facilitates future familiarity between the general practice and the pathology department and instils greater confidence in the mind of the practitioner in his or her future handling of cutaneous neoplasia.

In Scotland an additional component of the reporting of melanomas is their registration with the Scottish Melanoma Group. The first registration emanates from the pathologist, with a copy of this form being delivered to the local coordinator; this acts as an additional safeguard for recognising the lesion and confirming that appropriate management has been instituted.

\section{DIFFERENTIAI. DIAGNOSIS}

From the original pathology requests and from the questionnaire it appears that malignant melanoma was considered in the differential diagnosis in at most only $31 \%$ of cases, compared with $79 \%$ for hospital cases. Diagnostic accuracy for other skin malignancies excised by general practitioners has also been found to be low, ${ }^{2}$ but the clinical behaviour of malignan melanoma is different from that of other common skin malignancies. At present excision biopsies are being performed in the community on patients who are younger than the average patient with melanoma, including those with thick tumours, who have a poor prognosis. The dangers of malignant lesions being excised after incorrect diagnosis have been discussed, ${ }^{230131+}$ although attention has mostly been paid to basal cell carcinomas and squamous cell carcinomas, for which the implications of incomplete excision are very different from those of malignant melanoma. Despite the fact that some practitioners still argue against sending all specimens for histological examination, our results re-emphasise the need for histological examination of every excision biopsy specimen..$^{15}$

We believe that general practitioners should not be discouraged from excising pigmented lesions. Nevertheless, our results suggest that malignant melanoma is not being considered as the principal diagnosis to be excluded when many such lesions are excised.
Although much work has already been done to improve diagnostic accuracy by use of the revised seven point check list, ${ }^{16}$ further education is required to heighten the awareness of malignant melanoma among general practitioners and to provide further training for those general practitioners on the minor surgical list to increase the frequency with which pigmented lesions are adequately excised.

We thank the Scottish Melanoma Group for access to its database. We also thank all those general practitioners whom we contacted for their willing and prompt help with our questionnaire

1 Department of Health and W'elsh Office. General practice in the National Health Service: a new contract. London: Department of Health and Welsh Office, 1989

$2 \operatorname{Cox} \mathrm{NH}$, Wagstaff R, Popple AW. Using clinicopathological analysis of general practitioner skin surgery to determine educational requirements and guidelines. $B M 7$ 1992:304:1177-9.

3 McWilliam LJ, Knox F, Wilkinson N, Oogarah P. Performance of skin biopsies by general practitioners. BMF 1991;303:1177-9.

4 Hillan KJ, Johnson CP, Morton R. Effects of general practitioner contract on referral of specimens for histological examination. BMf 1991;303:1180.

5 Pitcher R, Gould DJ, Bowers DW. An analysis of the effects of general $\mathcal{G}$ practitioner minor surgery clinics on the workload of a district general hospital pathology and dermatology department. Br f Dermatol 1991; 125(suppl 38):93

6 Williams RB, Burdge AH, Lewis Jones S. Skin biopsy in general practice. $B M 7$ 1991;303:1179-80.

7 Ellwood JM Gallacher RP. The first signs and symptoms of melanoma: a population-based study. Pimuen Coll 1988:9:118-30.

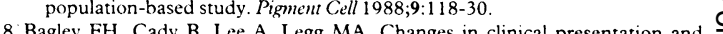
mey FH, Cady B, Lee A, Legg MA. Changes in clinical pres Rampen FHJ, Kint A, Hunter JAA, Hulsebosch H-J. Primary management of $\vec{N}$ melanoma. Br 7 Dermatol 1984;111:431-6.

10 Urist MM, Balch CM, Milton GW. Surgical management of the primary melanoma. In: Balch CM, Milton GW, eds. Cutaneous melanoma. Philadelphia: Lippincott, 1985:71-90.

11 Landthaler M, Braun-Falco O, Leitl A, Konz B, Holzer D. Excisional biopsy as the first therapeutic procedure versus primary wide excision of malignant melanoma. Cancer 1989;64:1612-6.

12 Lederman JS, Sober AJ. Does biopsy type influence survival in clinical stage 1 cutaneous melanoma? $\mathcal{A}$ Am Acad Dermatol 1985;13:983-7.

13 Shrank AB. General practitioners and minor surgery. $B r$ F Dermatol 1991:125: $599-600$

14 Whimster WF, Leonard RA. Surgical pathology in general practice. BMY 1991;303:1149-50.

15 Brazier JE, Lowy A. Performance of skin biopsies by general practitioners. BM7 1991;303:1472.

16 MacKie RM Malignant melanoma. A guide to early diagnosis. Glasgow: University of Glasgow, 1989

(Accepted 23 October 1992)

\section{ANY QUESTIONS}

What advances, if any, have been made in treating sulphite allergy?

Sulphiting agents, which are added to foods as preservatives or occur naturally as products of fermentation, notably in beers and wines, include sulphur dioxide and inorganic sulphites, which liberate sulphur dioxide under appropriate conditions. Although they are generally regarded as safe, ingested sulphites induce bronchospasm in $4-10 \%$ of people with asthma, most frequently women who are non-atopic

Inhalation of high doses $(0.5$ to $5 \mathrm{ppm})$ of sulphur dioxide induces bronchospasm in all people with asthma this is usually attributed to a cholinergic reflex via tracheobronchial receptors. Not all studies show inhibition of bronchoconstriction by anticholinergic drugs. ${ }^{1}$ The mechanisms by which ingested sulphites trigger bronchospasm have not been fully defined, although the rapid onset of bronchospasm and rapid relief with bronchodilators suggest that inhalation of released sulphur dioxide may occur.

Immediate hypersensitivity mediated by sulphite specific IgE may be detected, usually by skin testing, in a small proportion of patients who are sensitive to sulphite and who may also suffer rhinitis, angio-oedema, urticaria, or anaphylaxis after ingestion of sulphite.? Disodium cromoglycate has been shown to be effective in inhibiting sulphite induced bronchoconstriction.

Partial deficiency of sulphite oxidase, the enzyme responsible for the terminal oxidation of sulphite to inactive sulphate, has been suggested as a possible cause of sulphite intolerance in some cases. This led to the suggestion that cobalamins (vitamin $\mathrm{B}_{12}$ ), which are able to catalyse the extracellular non-enzymatic oxidation of sulphites, might prevent accumulation of sulphite and bronchospasm in people with asthma who are sensitive to sulphite. Premedication with a single dose of cyanocobalamin $(1500 \mu \mathrm{g})$ blocked the response to challenge with oral metabisulphite in four of five children in a single blind, placebo controlled study, an effect which persisted for at least four to 13 days.

Determining the relative importance of these differen pathogenetic mechanisms remains a challenge. Improved food labelling noting added sulphites would help patients in their efforts to avoid ingesting sulphite.-PENNY FITZHARRIS, consultant allergist, London

1 Nichol GM, Nix A, Chung KF, Barnes PJ. Characterisation of bronchoconstrictor responses to sodium metabisulphite aerosol in atopic subjects with and without asthma. Thorax 1989;44:1009-14.

2 Boxer MB, Bush RK, Harris KE, Patterson R, Pruzansky JJ, Yang WH. The laboratory evaluation of $\operatorname{IgE}$ antibody to metabisulphites in patien skin test positive to metabisulphites. I Allergy Clin Immunol 1988:82: skin test $622-6$.

3 Anibarro B, Caballero MT, Garcia-Ara MC, Diaz-Pena JM, Ojeda JA. Asthma with sulphite intolerance in children: a blocking study with cyanocobalamin. f Allergy Clin Immunol 1992;90:103-9. 